\title{
Treatment of dyslipidemia in the elderly
}

\author{
Hong Shao ${ }^{1}$, Li-Quan Chen $^{1}$, Jun $\mathrm{Xu}^{2}$ \\ ${ }^{1}$ Qingdao Municipal Hospital, 1 JiaoZhou Road, Qingdao 510630, China \\ ${ }^{2}$ Department of Biochemistry, University of Texas Southwestern Medical Center at Dallas, Dallas, TX 75390-9038, USA
}

\begin{abstract}
Dyslipidemia is a well-established risk factor for atherosclerosis. Treating dyslipidemia in elderly patients requires specific knowledge and understanding of common dyslipidemias and the relative safety of various pharmacologic agents in the presence of possible multiple comorbidities. Lifestyle modification remains the first step in the treatment of dyslipidemia; however, it can be difficult to sustain and achieve acceptable compliance in the elderly and it is best used in combination with drug therapy. Statins are widely accepted as the first-line therapy. Several recent studies have demonstrated that statins are safe and effective in the elderly. However, it is important to note that there is very limited data regarding the effects of dyslipidemia treatment on morbidity and mortality in patients over 85 years of age. In summary, the clinicians must recognize that the presence of dyslipidemia in the elderly poses substantial risk of coronary events and stroke. The available evidence has demonstrated that in most elderly patients who are at increased risk for cardiovascular morbidity and mortality, treatment of dyslipidemia with appropriate therapy reduces the risk, and when used carefully with close monitoring for safety, the treatment is generally well tolerated. With increasing life expectancy, it is critical for physicians to recognize the importance of detection and treatment of dyslipidemia in the elderly.
\end{abstract}

J Geriatr Cardiol 2011; 8: 55-64. doi: 10.3724/SP.J.1263.2011.00055

Keywords: dyslipidemia; atherosclerosis; statins

\section{Introduction}

China is facing a great challenge of population aging. By the end of 2005, there were 143 million Chinese people aged 60 and older. In addition, China's aging population has been growing rapidly, at a rate of $3.28 \%$ annually since 2001 and is expected to reach 437 million by $2051 .^{[1]}$

Along with the growing number of elderly population, the prevalence of cardiovascular disease is also increasing in China. ${ }^{[2,3]}$ It is well known that coronary artery disease is highly prevalent and leads to significant morbidity and mortality in the elderly. Approximately $80 \%$ of all death due to cardiovascular disease occurs in patients over 65 years of age. ${ }^{[4]}$ The high prevalence of coronary artery disease in the elderly is related to the fact that many of the well-established coronary risk factors exist in the elderly. Frequently, common risk factors, such as lipid abnormalities, diabetes, obesity and hypertension, are clustered together. A number of recent trials have demonstrated that the increased risk of

Correspondence to: Jun $\mathrm{Xu}, \mathrm{MD}$, Department of Biochemistry, University of Texas Southwestern Medical Center at Dallas, Dallas, TX 75390-9038, USA.Email: jxu99@hotmail.com

Received: July 7, 2010

Revised: December 12, 2010

Accepted: March 19, 2011

Published online: March 28, 2011 cardiovascular morbidity and mortality can be substantially reduced by appropriate risk factor modification. Although the benefit of treatment of hypertenison in the elderly is well established, there is still considerable debate regarding the need for treatment of dyslipidemia in the elderly at risk. In this review, the authors discuss the relevance of hyperlipidemia in the elderly and primarily focus on the rationale for the treatment of dyslipidemias in these patients.

\section{Lipid abnormalities and subclinical atherosclerosis in the elderly}

Total cholesterol has been found to be highest in the sixth decade of life and then falls slightly with advancing age. Patients over 65 years of age have a high prevalence of hyper-lipidemia, with total cholesterol levels greater than $240 \mathrm{mg} / \mathrm{dL}$ in approximately $25 \%$ of men and $42 \%$ of women. ${ }^{[2]}$ The Chinese National Nutrition and Health Survey (CNHS) in 2002 showed the mean TC level was $4.21 \mathrm{mmol} / \mathrm{L}$ in those over 60 years, while it was 3.70 $\mathrm{mmol} / \mathrm{L}$ and $4.09 \mathrm{mmol} / \mathrm{L}$, respectively, in those aged 18 44 years and $45-59$ years. ${ }^{[5]}$ One of the limitations in the evaluation of the association between cholesterol level and coronary heart disease comes from the different methodologies used to estimate the risks in elderly patients. The traditional

\footnotetext{
http://www.jgc301.com; jgc@mail.sciencep.com | Journal of Geriatric Cardiology
} 
measure is relative risk, which is the ratio of the incidence of coronary heart disease at high cholesterol levels to the incidence at low cholesterol levels, typically calculated over 5-10 years. Due to the fact that coronary heart disease is common in older patients, the relative risk between cohorts with high- and low-cholesterol values diminishes with increasing age. ${ }^{[6]}$

Excess absolute (attributable) risk is a more accurate measure in older patients. Absolute risk estimates the likelihood that a person will develop coronary heart disease within a pre-specified time. Attributable risk defines the difference in absolute risk between groups with high and low cholesterol levels. ${ }^{[7,8]}$ Attributable risk can also provide an estimate of how much the risk can be lowered if cholesterol levels are reduced. ${ }^{[8]}$ With use of a mathematical model on the data from the Framingham Heart Study, for each risk factor the risk of coronary heart disease increased continuously with age and the highest was in hypertensive smokers with elevated total cholesterol and high-density lipoprotein (HDL) cholesterol levels of $35 \mathrm{mg} / \mathrm{L}$ and the lowest was in normotensive nonsmokers with average total cholesterol and HDL cholesterol levels. ${ }^{[9]}$ Data from the Honolulu Heart Study also highlights the usefulness of attributable risk assessments in older subjects and confirms that elevated cholesterol has a greater impact on the development of coronary heart disease in older men than in the middle-aged and strengthens the case for screening and intervention in older patients with hypercholesterolemia. ${ }^{[10]}$

Even in the elderly who do not have overt heart disease, it is important to consider subclinical atherosclerosis when the absolute risk is high and benefit of the treatment is more rewarding. The prevalence of subclinical atherosclerosis in adults 65 years of age or over was evaluated in the Cardiovascular Health Study (CHS) and was found to be more than $30 \%$, which increased further with advancing age. ${ }^{[11]}$ Using intravenous ultrasound on the donor hearts, it was demonstrated that subclinical disease can be as high as $85 \%$ in patients over 50 years of age. ${ }^{[12]}$ In most of the primary and secondary prevention trials, cholesterollowering therapy reduced the risk of cardiovascular events to a similar extent in the older ( $\geq 65$ years) and younger individuals. Older patients, who had previous history of coronary artery disease and mildly elevated cholesterol, had more absolute risk reduction from treatment compared with younger patients in the combined cardiovascular risk and all-cause mortality. ${ }^{[13]}$ Based on the results of a number of randomized clinical trials, it is evident that the treatment of dyslipidemia in the elderly is an effective means to reduce the risk of cardiovascular events in those who are at intermediate-to-high risk. Furthermore, it is important to note that in the elderly, the benefit of treatment of dyslipidemia might be evident as early as the first 2 years of therapy.

Aging dyslipidemia is becoming more prominent in women than in men, which has important clinical relevance due to the fact that even though it is not well recognized, women 65 years of age or over have a higher coronary event rate. ${ }^{[14]}$ A possible explanation for the increase in lipid abnormalities in the elderly women is that with advancing age and menopause the levels of HDL cholesterol decreases, making the gender difference in dyslipidemia less prominent as in younger age groups. HDL cholesterol plays an important role in the risk stratification and prognosis of cardiovascular events. The prognostic value of the HDL cholesterol in the elderly was prospectively evaluated in the CHS, which examined the association between lipid levels and cardiovascular events in older adults. In the CHS, a total of 5201 adults 65 years of age and older had fasting lipid levels measured and were followed for an average of 7.5 years. The results of this study demonstrated a strong association between low levels of HDL cholesterol and increased risk of myocardial infarction. ${ }^{[15]}$

The dramatic decline in coronary artery disease in the elderly in the USA for the last 30 years emphasizes the potential for coronary event reduction and cardiovascular disease prevention in this population. ${ }^{[16]}$ Although some of the risk factors in the elderly, such as gender, age and familial predisposition, cannot be changed, there are many other modifiable risk factors. These include physical inactivity, obesity, smoking, diabetes, hypertension and dyslipidemia, which can be modified with therapeutic lifestyle changes and pharmacologic interventions. Therapeutic lifestyle changes are highly effective; however, they are difficult to sustain for a long period of time and usually have a major role as a complimentary treatment to drug therapy. Pharmacologic approaches for the treatment of hypertension have proven to be very effective in reducing the risk of cardiovascular events in the elderly. Recent data from a number of clinical trials also demonstrate that treatment of hyperlipidemia in the elderly is also beneficial, especially in those at higher risk of coronary events. Treating dyslipidemia in the elderly requires a comprehensive approach, which should include evaluation of the existing risk factors for cardiovascular disease, including global risk assessment with the available tools and setting of the appropriate target goals for lipid profile.

\section{Lifestyle modifications for the elderly}

As emphasized by the National Cholesterol Education Program (NCEP) Expert Panel on Detection, Evaluation, and Treatment of High Blood Cholesterol in Adults [Adult 
Treatment Panel (ATP)-III], guidelines for the initial approach in all patients with dyslipidemia should be therapeutic lifestyle changes, which need to include not only dietary modifications but also increase in physical activity. The Centers for Disease Control and Prevention and the American College of Sports Medicine have reported that up to $24 \%$ of elderly people are completely sedentary and $54 \%$ are inadequately active. ${ }^{[17]}$ The NCEP/ATP-III guidelines recommend an aerobic exercise regimen for all patients with elevated cholesterol levels that are physically able to follow an individually tailored program. Cardiac rehabilitation and exercise training programs which included elderly patients with coronary heart disease were proven to produce a modest but statistically significant improvement in plasma lipids, with approximately 5\% reduction in total cholesterol, $15 \%$ reduction in triglycerides, $3 \%$ reduction in low-density lipoprotein (LDL) cholesterol and 6\% increase in HDL cholesterol. ${ }^{[17]}$ In certain subgroups of elderly patients with very low HDL cholesterol and hypertriglyceridemia, greater increases in HDL cholesterol of up to $15 \%$ were achieved. ${ }^{[18]}$ These positive effects of exercise on dyslipidemia can be additive to pharmacotherapy and also help improve the general functional status and overall quality of life in elderly individuals.

An important element of therapeutic lifestyle changes includes modification of the diet. In the past, the American Heart Association had recommended low-calorie, low-fat diets, with high-carbohydrate content; however, this kind of diet can lead to elevated triglycerides and further reduce HDL cholesterol. Although the clinical relevance of these changes is not clear, such effects can be harmful in those with elevated LDL cholesterol. Due to these concerns, there is a growing interest to reduce the amount of carbohydrate in the diet and increase the protein intake. In one study of 63 obese patients, the low carbohydrate diet (Atkins ${ }^{\circledR}$ diet) was found to produce a greater weight loss for the first 6 months but there was no difference at one year compared with conventional diets. ${ }^{[19]}$ The same findings were demonstrated in another study comparing Orinish, Atkins, Weight Watchers and Zone Diets. ${ }^{[20]}$ It was also demonstrated that HDL cholesterol was increased more with a low-carbohydrate diet that with a low-fat regimen. ${ }^{[21]}$ These studies did not involve a large number of elderly patients and further trials are needed to evaluate the effects of these diets on the elderly. In general, the long-term effect of a low-fat diet is relatively minor and will lead to only a $2 \%-5 \%$ reduction of LDL cholesterol. It is prudent to avoid diets which reduce HDL cholesterol due to its cardioprotecive role. ${ }^{[22,23]}$ Substituting soy-derived protein for animal protein reduces LDL cholesterol and triglycerides. ${ }^{[23]}$
Based on these considerations, it is now recommended that individuals with dyslipidemia should reduce intake of saturated fat, limit the consumption of simple carbohydrates and increase the proportion of protein in their diet. ${ }^{[22,24,25]}$ Recently, it has also been demonstrated that both plant- and marine-derived omega- 3 fatty acids reduce the incidence of coronary heart disease. The omega- 3 fatty acids from fish oil, if consumed in the dose of $4 \mathrm{~g} / \mathrm{d}$, can reduce triglycerides by $25 \%-30 \%$, with accompanying mild elevations of LDL cholesterol by $5 \%-10 \%$, and increase HDL cholesterol by $1 \%-3 \%$. The triglyceride lowering effect of the omega-3 fatty acids is dose related. ${ }^{[25]}$ Additional research is needed to define the precise role and the appropriate dose of omega-3 fatty acids in the management of dyslipidemia in elderly patients. Recent studies have also demonstrated that replacement of fat with plant stanol esters (e.g., Benecol ${ }^{\circledR}$ margarine), in patients with mild hyperlipidemia, can be used as an adjunct to statin therapy and can typically produce additional $10 \%-15 \%$ reduction in LDL cholesterol. This dietary intervention has been considered cost-effective and can be particularly helpful in patients not reaching their goal with statin therapy and where an increase in statin dose might have little additional benefit and could be associated with more side effects. Another dietary intervention which has been proven to be effective is addition of soluble fibers as a cholesterol-lowering agent. Elderly patients are particularly predisposed to constipation and colon disease, and the use of soluble fiber will improve these symptoms in parallel with reduction in LDL cholesterol by up to $20 \%$ (usually $5 \%-10 \%$ ), without significant impact on HDL cholesterol levels.

The benefits of good dietary habits were emphasized in a study of 2339 apparently healthy men and women 70-90 years of age, conducted in 11 European countries. The study revealed that adherence to a Mediterranean diet and healthy lifestyle is associated with more than 50\% reduction in all-cause as well as cardiovascular mortality. ${ }^{[21]}$ Furthermore, the therapeutic lifestyle changes may provide additional benefit in the elderly, based on the data from the Third National Health and Nutrition Examination Survey (19881994), which revealed that the prevalence of metabolic syndrome is in excess of $40 \%$ in the elderly. ${ }^{[26]}$ The presence of metabolic syndrome in the elderly is particularly relevant because they have confluence of multiple cardiovascular risk factors. For patients over 65 years of age, lifestyle modifications could be the most difficult to achieve. Patients may become depleted in the major nutritional elements, such as minerals and protein, if they are not educated appropriately regarding the necessary dietary changes. The emphasis on the diet rich in protein and 
restricted in carbohydrates, although the most effective treatment for metabolic syndrome, is lifestyle modification; an aggressive approach with pharmacologic agents, especially for dyslipidemia, might be needed in many elderly individuals.

\section{Pharmacologic approach to dyslipidemia in the elderly}

The decision to treat dyslipidemia in the elderly as well as the target lipid levels should be based on the current NCEP/ATP III Expert Panel recommendations. ${ }^{[27]}$ It is difficult to distinguish between primary versus secondary prevention in the elderly, due to the high prevalence of subclinical atherosclerosis. In addition, the absolute risk of cardiovascular events in the elderly is quite high, particularly when there is presence of subclinical atherosclerosis, which can be diagnosed by carotid ultrasound, ankle-brachial index and echocardiographic or electrocardiographic abnormalities. ${ }^{[28]}$ Therefore, lipid-lowering therapy should be considered in the elderly, not only when there is the presence of clinical disease, but also when they have subclinical atherosclerosis and are at increased risk as estimated by global risk assessment. Although several choices are available for the treatment of dyslipidemia, statins constitute the primary pharmacologic approach in most elderly patients.

\section{Statins}

The statins are considered the first-line therapy for lowering LDL cholesterol in the elderly, based on the findings from a number of studies, including the Scandinavian Simvastatin Survival Study (4S), Cholesterol and Recurrent Events (CARE), Long-term Intervention with Pravastatin in Ischemic Disease (LIPID), Air Forse/Texas Coronary Atherosclerosis Prevention Study (AFCAPS/ TexCAPS), Prospective Study of Pravastatin in the Elderly at Risk (PROSPER), Heart Protection Study (HPS) and Anglo-Scandinavian Cardiac OUTCOMES Trial-LipidLowering Arm (ASCOT-LLA. ${ }^{[28-35]}$ Overall, the result of these studies demonstrated that treatment with statins reduces cardiovascular events and all-cause mortality and is safe and well-tolerated by the elderly. Some dissimilarities in the structure, potency, lipophilicity and pharmacokinetics exist among the statins, although they have the same lipidmodifying effect through a partial and reversible reduction in the activity of 3-hydroxy-3-methylglutaryl (HMG)-CoA reductase, which decreases cholesterol synthesis. The choice of statin should be guided by the goals of therapy and effects on LDL cholesterol, HDL cholesterol and triglycerides in the elderly.

The statins have been found to have multiple indirect effects on vasculature, in addition to their direct LDL cholesterol-lowering effect. These additional actions are commonly referred to as pleiotropic effects, which include those on endothelial function, vascular inflammation, oxidative stress, thrombosis and platelet aggregation and adhesion of platelets and white cells to the vascular endothelium. These pleiotropic effects are thought to result in the stabilization of the vulnerable plaque(s) and promote new vessel formation. ${ }^{[36]}$ Although not proven, these actions of statins may play a role in reducing the risk of cardiovascular events. Results of the Myocardial Ischemia Reduction with Aggressive Cholesterol Lowering (MIRACL) study suggested the potential role of pleiotropic effects of statins, by demonstrating a reduction in the rate of recurrent ischemic events with atorvastatin after acute coronary syndrome, as early as 6-8 wk after treatment initiation. ${ }^{[37]}$

Statins have also been found to reduce the risk of deep venous thrombosis and possibly the risk of dementia, which makes this group of drugs even more relevant in the management of the elderly. ${ }^{[38-40]}$ In addition, there is an observational study suggesting that statins may reduce the risk of fractures, which are well known to occur frequently and increase the mortality in the elderly. ${ }^{[41]}$

The PROSPER is the only prospective study which has specifically examined the role of lipid-lowering therapy with statins in the elderly. The primary hypothesis of PROSPER was that pravastatin will reduce myocardial infarction and stroke events in elderly patients with existing vascular disease or at significant risk of developing vascular events. This randomized, double-blind, placebo-controlled trial included 2804 men and 3000 women 70-82 years of age with a history of, or risk factors for, vascular disease. Pravastatin $40 \mathrm{mg} / \mathrm{d}$ was administered to 2891 subjects and a matching placebo was given to 2913. Follow-up was 3.2 years on average and primary end point was a composite of coronary death, nonfatal myocardial infarction and fatal or nonfatal stroke. The results revealed that pravastatin lowered LDL cholesterol concentrations by 34\%. Mortality from coronary disease fell by $24 \%$ in the pravastatin group. This study provides evidence that older people between 70 and 82 years of age can reduce their risk of dying from coronary heart disease by almost a quarter and of having a myocardial infarction by almost a fifth through treatment with cholesterol-lowering pravastatin for 3 years. ${ }^{[33]}$

The heart protection study also involved a large number of individuals above 65 years of age and demonstrated not only significant reduction in coronary events in patients 
taking simvastatin $40 \mathrm{mg}$, but also in the cerebrovascular events, despite the lack of any demonstrated relationship of stroke incidence to blood lipids. ${ }^{[42]}$

There are also smaller studies which have evaluated the benefit of treatment with the statins in the special population of elderly patients with renal insufficiency. It is well known that patients with renal disease are at high risk of cardiovascular events, though this increased risk could be related to factors leading to renal disease (e.g., hypertension and diabetes) and not the disease itself. Specifically, the elderly, even with mild chronic renal failure and microalbuminuria, have been demonstrated to have increased susceptibility to cardiovascular events. This increased risk is comparable with the risks related to diabetes and left ventricular hypertrophy. ${ }^{[43]}$ The similarities between atherosclerosis and glomerulosclerosis (both involving endothelial dysfunction) lead to the concept that the benefits of statins can be applied to the prevention of progression of renal disease. It could be postulated that by using statins in patients with chronic renal failure, nephroprotection could be achieved along with cardioprotection. ${ }^{[4]}$

\section{Safety of the statins in the elderly}

The use of statins in the elderly is generally considered safe. However, following the withdrawal of cerivastatin, there is increasing concern regarding some of the side effects of the statins, particularly, drug-induced myopathy and elevated liver enzymes. During the past 10 years, several large-scale randomized clinical trials have evaluated the effects of the statins on liver enzymes, and revealed that all can be associated with some degree of transaminase elevation, although the incidence is generally less than $2 \%$ (when toxicity is defined as an elevation of liver enzymes in excess of three-times the upper limits of normal). The enzyme elevation most commonly develops within the first 4-12 mo from the start of the treatment and it is generally recommended that the liver function should be monitored during that time. ${ }^{[45-51]}$ In the Statin Therapies for Elevated Lipid Levels Compared Across Dose Ranges to Rosuvastatin (STELLAR) trial, it was demonstrated that the alteration of hepatic function associated with statin therapy appears to be dose related, although only small absolute differences in the incidence of elevated transaminases were noted with increasing doses. ${ }^{[47]}$

Clinically significant myopathy is uncommon with statin therapy; however, rabdomyolisis can develop in $0.1 \%$ of patients. ${ }^{[48]}$ In the elderly, the highest incidence of myopathy occurs when coexisting disease is present, for example, renal insufficiency.
The mechanisms of the statin-induced myotoxicity has not been clearly elucidated. In vitro studies have implicated a statin-induced depletion of metabolic intermediates in the cholesterol synthetic pathway, including mevalonate, farnesol and geranylderaniol, as a potential cause of myopathy, as when these compounds were repleated myopathy was reduced. ${ }^{[49]}$

All of the available statins, with the exception of pravastatin and rosuvastatin, are metabolized by the cytochrome P450 (CYP) system. Serum concentrations of these statins can potentially be increased when other medications competing for the CYP system are prescribed and can lead to increased risk of myositis and rhabdomyolysis. The same problem can develop when statin interacts with food containing the CYP isoenzyme inhibitor, particularly grapefruit juice, frequently consumed by the elderly. ${ }^{[50]}$ The risk of drug interaction is especially high in the elderly due to coexistence of multiple comorbidities and the need for polypharmacy. The possibility of myositis with the use of statins is increased, especially when statins are used in combination with a fibrate, cyclosporin, erythromycin, nicotinic acid, the protease inhibitors, nefazodone and macrolide antibiotics. ${ }^{[49]}$

Although uncommon, some patients can develop insomnia, bad or vivid dreams and difficulty sleeping or concentrating. For these patients, statin with low penetration in the CNS, such as pravastatin, might be preferred. ${ }^{[51,52]}$ A recently published study demonstrated minor decrements in cognitive functioning with statins and the long-term effects of these changes remains unknown. ${ }^{[53]}$

It is also important to note that advanced age can be associated with reduced clearance of the statin and its metabolites, which in combination with other drugs affecting the same clearance mechanisms, can increase the probability of adverse effects. Therefore, it is advisable to start the statin in lower doses in elderly patients and use it in combination with as few concomitant drugs as possible. Many elderly patients may use some over-the-counter medications; however, they should be educated and cautioned regarding the potential drug interactions with prescribed statin. ${ }^{[54]}$ Overall, the incidence of serious adverse events associated with the use of statins is extremely low and the discontinuation rate due to significant adverse events is below $10 \%{ }^{[55]}$

Despite the popularity and undisputable benefits of statins, there may be clinical situations in which treatment with other lipid-lowering agents, such as fibrates, may be indicated. 


\section{Fibrates}

The major benefit of the fibrate class of drugs (gemfibrozil, clofibrate and fenofibrate) is their effects on triglicerides and HDL cholesterol. As with other age groups, low serum HDL cholesterol is also considered to be a risk factor for cardiovascular disease in the elderly. ${ }^{[14]}$ Fibrates are frequently used in patients who have low HDL cholesterol and/or high triglycerides. The mechanism of action involves activating the nuclear peroxisome proliferator-activated receptor (PPAR)- $\alpha$, resulting in an increased production of lipoprotein lipase, which promotes rapid very-low-density lipoprotein (VLDL) cholesterol turnover, leading to a decrease in triglycerides. Fibrates also induce the diversion of hepatic free fatty acids from esterification reactions to those of oxidation, thereby decreasing the liver's secretion of triacylglycerol- and cholesterol-rich VLDL cholesterol. Fibrates also have direct antiatherogenic effects on vascular tissue by inhibiting tumor necrosis factor- $\alpha$, which induces expression of vascular cellular adhesion molecules by the endothelium. ${ }^{[49,50]}$

The fibrates are considered to be highly effective triglyceride-lowering drugs, producing as much as $50 \%$ reduction $(25 \%-30 \%$ in patients with lower levels of triglycerides) and an 11\%-14\% increase in HDL cholesterol (increase of $>25 \%$ can occur in patients with hypertriglyceridemia and low HDL cholesterol) with approximately $11 \%$ decrease in LDL cholesterol. ${ }^{[17,51,55]}$ Currently, no large trials have examined the effects of fibrate therapy on improvement in lipids or cardiovascular event reduction in the patients over 75 years of age. In younger patients, the risk of myocardial infarction was reduced by $9 \%-34 \%$ in four large clinical studies: World Health Organization Clofibrate Study, Helsinki Heart Study (HHS), Bezafibrate Infarction Prevention (BIP) and Veterans Affairs Program High-Density Lipoprotein Cholesterol Intervention Trial (VA-HIT). ${ }^{[51,56-59]}$ Clofibrate and fenofibrate were found to cause fewer gastrointestinal symptoms than gemfibrozil and could be preferable in elderly patients with underlying gastrointestinal disorders, although the overall reported incidence of gastrointestinal problems with fibrates is approximately $5 \%$. It should be noted that clofibrate can cause erectile dysfunction.

Although gemfibrozil is cheaper and has been demonstrated to be effective in reducing the clinical event rate in two major clinical studies, it has minimal to no effect on lowering LDL cholesterol and a higher risk of drug interactions with statins, especially those which require metabolism by CYP or require glucoronidation, as described above. ${ }^{[16,57,59]}$ For example, fibrates have been demonstrated to displace warfarin from protein-binding sites and may thus require modification of the dose. Interactions such as this are clinically important due to the frequent use of concomitant drugs in the elderly. Of the currently available fibrates, fenofibrate is considered to be the preferred agent, due to once-daily dosing, a favorable adverse reactions profile (especially when prescribed with statins), slightly increased potency in lowering triglycerides and raising HDL cholesterol, and lowering LDL cholesterol. All fibrates are excreted by the kidneys and can accumulate in serum in patients with renal failure, which can lead to myositis; therefore, in the elderly patients with reduced renal function, the dose should be adjusted accordingly to avoid toxicity.

\section{Nicotinic acid}

Nicotinic acid is an essential water-soluble B-complex vitamin, which has also demonstrated a hypolipidemic effect independent of its role as a vitamin, although a higher pharmacologic dose is required to achieve this effect. Nicotinic acid inhibits the mobilization of free fatty acids from peripheral tissue, thereby reducing hepatic synthesis of triglycerides and secretion of VLDL cholesterol and its conversion to LDL cholesterol.

Unfortunately, in the elderly population, as well as among younger patients, this agent is underutilized. Nicotinic acid can induce improvements in all aspects of the lipid profile, including lowering total and LDL cholesterol by $15 \%$, reducing triglycerides by $25 \%-30 \%$ and increasing HDL cholesterol between $25 \%$ and $40 \%$ in patients with low HDL cholesterol. In general, nicotinic acid is considered to be the best medication for treatment of low HDL cholesterol.

Based on the current evidence, the European Consensus Panel recommends that the minimum target for $\mathrm{HDL}$ cholesterol should be $40 \mathrm{mg} / \mathrm{dL}$ in patients with metabolic syndrome and it could be applied to elderly patients with high cardiovascular risks. ${ }^{[60]}$ Lipoprotein (a) is considered to be an additional cardiovascular risk factor. Nicotinic acid is also the best medication to reduce lipoprotein (a), by 30\%. ${ }^{[61]}$ This drug has another unique property: it can improve LDL cholesterol subclasses by changing LDL cholesterol's pattern from small, dense particles, which is known as a pattern $\mathrm{B}$ and considered more atherogenic, to larger, more buoyant particles (pattern A). This effect could be clinically important in the treatment of the elderly patients meeting criteria for metabolic syndrome. ${ }^{[62]}$ The possibility of worsening glucose intolerance with nicotinic acid in patients with metabolic syndrome or diabetes mellitus was one of the barriers for the use of this medication; however, this was 
not a significant limitation, as demonstrated by a recent study showing that lipid-modifying doses of nicotinic acid in patients with diabetes did not lead to a deterioration of glycemic control over a 60 -week period. ${ }^{[63]}$

It is also postulated that in addition to the benefits of nicotinic acid therapy, treatment with nicontinic acid can also reduce fibrinogen levels with the potential of reducing thrombotic events. ${ }^{[64]}$

The majority of side effects related to the use of nicotinic acid in the elderly have no significant clinical sequelae. The administration of aspirin $325 \mathrm{mg}, 30-60 \mathrm{~min}$ before each dose of nicotinic acid for a few days, taking nicotinic acid at the end of a meal and not with hot liquids, can minimize the flushing of the skin, which $10 \%$ of patients find intolerable ${ }^{[45]}$ The starting dose is $250-500 \mathrm{mg} / \mathrm{d}$ and should be increased monthly by $500-1000 \mathrm{mg} / \mathrm{d}$ to a maximum of $3000 \mathrm{mg} / \mathrm{d}$. Hepatitis is more frequent in patients taking nicotinic acid than in those taking statins, especially in 2000-3000-mg doses. Mild elevation of liver enzymes does not require discontinuation of the medication but close clinical observation is warranted due to fulminant hepatic necrosis and death cases associated with nicotinic acid. ${ }^{[64]}$ Other side effects include conjunctivitis, nasal stuffiness, loose bowel movements or diarrhea, acanthosis nigricans and ichthyosis. ${ }^{[54]}$ Proper dose adjustment and patient education can increase the compliance in elderly patients with nicotinic acid treatment.

\section{Bile acid sequestrants (resins)}

Bile acid-binding resins are now largely used as adjuncts to statin therapy, especially in patients for which additional reduction in LDL cholesterol by $10 \%-20 \%$ is desired and the level of triglycerides is not elevated. Currently available bile acid resins include cholestyramine, colesevelam and colestipol. These medications are usually administered in doses of 4-10 g twice a day with meals, as a suspension in juice or water. The increase in triglyceride concentrations induced by bile acid resins can be a problem, especially in patients prone to hypertriglyceridemia. Due to their mechanism of action, which is based on binding to bile acids in the small intestine, they lead to interruption of the enterohepatic circulation of bile acids and increase the conversion of cholesterol to bile in the liver.

These agents can inhibit the intestinal absorption of fat-soluble vitamins, including vitamin $\mathrm{D}$, warfarin, digoxin, levothyroxin, thiazide diuretics, folic acid and statins, which requires specific consideration in the elderly patients who very frequently require thyroid supplements, thiazide diuretics or vitamin $\mathrm{D}$ for osteoporosis.
In addition, up to $30 \%$ of patients will develop abdominal fullness, gas and constipation while taking bile acid resins, which could be corrected with dose adjustment and the use of fiber or prune juice in the daily diet.

\section{Ezetimibe}

Ezetimibe is the first of a new class of lipid-lowering drugs known as intestinal cholesterol absorption inhibitors. It could be administered in once-daily doses of $10 \mathrm{mg}$ and may reduce LDL cholesterol by $15 \%-20 \%$ as monotherapy and by $20 \%-25 \%$ when added to statin. ${ }^{[65]}$ The coadministration of ezetimibe with statins offers a well-tolerated and efficacious treatment of lowering LDL cholesterol in patients with metabolic syndrome and diabetes, and could be applied to the aged high-risk population. ${ }^{[59]}$ In addition, ezetimibe slightly raises HDL cholesterol and reduces levels of triglycerides by approximately $10 \%$. The combination of statin and ezetimibe is usually well tolerated but may occasionally result in a small increase in the incidence of elevated liver enzyme levels, although cases of severe hepatotoxicity have not been demonstrated.

An additional action of ezetimibe on lowering C-reactive protein may be another favorable indication to prevent cardiovascular disease in the elderly. ${ }^{[66-68]}$

\section{Conclusions}

It is well known that a majority of coronary events occur in the elderly and are associated with high mortality rates. With advancing age, there is an increase in the tendency to have atherogenic dyslipidemia, including elevations in LDL cholesterol levels. However, there is limited data correlating the increase in serum LDL cholesterol with the development of coronary events in the elderly. The emerging evidence during the past 10 years from a number of clinical trials suggests that statin therapy lowers the risk of coronary events in the elderly. Critical review of these clinical trials demonstrates that the benefit of lipid-lowering therapy, mainly LDL cholesterol, is the same or even greater in the elderly patients when compared with the younger ones. Lipid lowering is accomplished by therapeutic lifestyle modifications and drug therapy.

As with younger patients, treatment in the elderly should also begin with lifestyle and dietary modifications. Pharmacologic therapy, specifically with statins, is usually needed to achieve target LDL cholesterol and can be used safely in the elderly population to reduce the morbidity and mortality from cardiovascular disease. Lipid-lowering 
therapy in the elderly can have a substantial financial impact by reducing Medicare expenses, due to reductions in the number of coronary events and the need for revascularization therapy. To date, the cost-effectiveness and cost-saving benefits for secondary prevention of cardiovascular disease in the elderly patients have been demonstrated in two studies. ${ }^{[69,70]}$ In general, lipid-lowering therapy should be focused on the recently recommended goals, which depend on the global risk assessment. Based on currently available evidence, pharmacologic treatment should be started using statins as the first-line agent in the elderly population. In addition, a combination therapy including ezetimibe, niacin and fibrates is an effective modality for elderly patients when needed and can be safely used for primary or secondary prevention. In the older patients, the distinction between these two preventive measures is often difficult due to the high incidence of subclinical atherosclerosis in the elderly.

In patients over 75 years of age, a thorough assessment of overall health and comorbidities should be performed prior to initiation of any lipid-lowering therapy. The recent data confirms that more aggressive therapy is needed in high-risk patients to achieve the LDL cholesterol goal of below $70 \mathrm{mg} / \mathrm{dL}$ and a large proportion of the elderly are in this high-risk category of patients. An extra effort is required to promote the recent evidence supporting aggressive lipid management in the elderly. ${ }^{[71]}$

\section{References}

1 China National Committee on Aging: Announcement (in Chinese) [on-line]. http://www.cnca.org.cn (accessed August 2010).

2 Moran A, Gu D, Zhao D, et al. Future cardiovascular disease in China: markov model and risk factor scenario projections from the coronary heart disease policy model-China. Circ Cardiovasc Qual Outcomes 2010; 3: 243-252.

3 Zhang XH, Lu ZL, Liu L. Coronary heart disease in China. Heart 2008; 94: 1126-1131.

4 Dalal D, Robbins J. Management of hyperlipidemia in the elderly population: an evidence-based approach. South Med J 2002; 95: 1255-1261.

5 Zhao WH, Zhang J, Zhai Y, et al. Blood lipid profile and prevalence of dyslipidemia in Chinese adults. Biomed Environ Sci 2007; 20: 329-335.

6 Hall KM, Luepker RV. Is hypercholesterolemia a risk factor and should it be treated in the elderly? Am J Health Promot 2000; 14: 347-356.

7 Deedwania PC. Hypercholesterolemia. Is lipid-lowering worthwhile for older patients? Geriatrics 2000; 55: 22-28.

8 Grundy SM, Cleeman JI, Rifkind BM, et al. Cholesterol lowering in the elderly population. Arch Intern Med 1999;
159: $1670-1678$.

9 Ulrich S, Hingorani AD, Martin J, et al. What is the optimal age for starting lipid-lowering treatment? A mathematical model. BMJ 2000; 320: 1134-1140.

10 Benfante R, Reed D, Frank J. Do coronary heart diseas risk factors measured in the elderly have the same predictive roles as in the middle-aged. Comparisons of relative and attributable risks. Ann Epidemiol 1992; 2(3): 273-282.

11 Kuller L, Borhani N, Furberg C, et al. Prevalence of subclinical atherosclerosis and cardiovascular disease and association with risk factors in the Cardiovascular Health Study. Am J Epidemiol 1994; 139: 1164-1179.

12 Tuzcu EM, Kapadia SR, Tutar E, et al. High prevalence of coronary atherosclerosis in asymptomatic teenagers and young adults. Circulation 2001; 103: 2705-2710.

13 Hunt D, Young P, Simes J, et al. Benefits of pravastatin on cardiovascular events and mortality in older patients with coronary heart disease are equal to or exceed those seen in younger patients: results from the LIPID trial. Ann Intern Med 2001; 134: 931-940.

14 Kannel WB. Coronary heart disease risk factors in the elderly. Am J Geriatr Cardiol 2002; 11: 101-107.

15 Psaty BM, Anderson M, Kronmal RA, et al. The association between lipid levels and the risks of incident myocardial infarction, stroke, and total mortality: The Cardiovascular Health Study. J Am Geriatr Soc 2004; 52: 1639-1647.

16 Wenger NK. Dylipidemia as a risk factor at elderly age. Am J Geriatr Cardiol 2004; 13 (Suppl. 1): 4-9.

17 Pate R, Pratt M, Blair S, et al. Physical activity and public health. A recommendation from the Centers for Disease Control and Prevention and the American College of Sports Medicine. JAMA 1995; 273: 402-407.

18 Lavie CJ. Treatment of hyperlipidemia in elderly persons with exercise training, nonpharmacologic therapy and drug combination. Am J Geriatr Cardiol 2004; 13 (3, Suppl. 1): 29-33.

19 Foster GD, Wyatt HR, Hill JO, et al. A randomized trial of a low carbohydrate diet for obesity. N Engl J Med 2003; 348: 2082-2090.

20 Shai I, Schwarzfuchs D, Henkin Y, et al. Weight loss with a low-carbohydrate, Mediterranean, or low-fat diet. $N$ Engl $J$ Med 2008; 359: 229-241.

21 Yancy WS Jr, Olsen MK, Guyton JR, et al. A low carbohydrate, ketogenic diet versus a low fat diet to treat obesity and hyperlipidemia: a randomized, controlled trial. Ann Intern Med 2004; 140: 769-777.

22 Bravata DM, Sanders L, Huang J, et al. Efficacy and safety of low carbohydrate diets: a systemic review. JAMA 2003; 289: 1837-1850.

23 Merritt JC. Metabolic syndrome: soybean foods and serum lipids. J Natl Med Assoc 2004; 96: 1032-1041.

24 Aude YW, Mego P, Mehta J. Metabolic syndrome: dietary interventions. Curr Opin Cardiol 2004; 19: 453-459.

25 Knoops KT, de Groot LC, Kromhout D, et al. Meditarian diet, 
lifestyle factors, and 10-year mortality in elderly European men and women. The HALE project. JAMA 2004: 292: 1433-1439.

26 Ford ES, Giles WH, Dietz WH. Prevalence of metabolic syndrome among US adults: findings from the third National Health and Nutrition Examination Survey. JAMA 2002; 287: 356-359.

27 Executive Summary of the Third Report of the National Cholesterol Education Program (NCEP) Expert Panel on Detection, Evaluation, and Treatment of High Blood Cholesterol in Adults (Adult Treatment Panel III). JAMA 2004; 285: 2486-2497.

28 Garcia-Palmiery M. Control of blood lipids in the elderly. Bol Asoc Med PR 2003; 95: 84-90.

29 Pedersen TR. Coronary artery disease: the Scandinavian Simvastatin Survival Study experience. Am J Cardiol 1998; 82: 53T-56T.

30 Sacks FM, Pfeffer MA, Moye LA, et al. The effect of pravastatin on coronary events after myocardial infarction in patients with average cholesterol levels. Cholesterol and Recurrent Events Trial investigators. N Engl J Med 1996; 335: 1001-1009.

31 The Long-Term Intervention with Pravastatin in Ischaemic Disease (LIPID) Study Group. Prevention of cardiovascular events and death with pravastatin in patients with coronary heart disease and a broad range of initial cholesterol levels. $N$ Engl J Med 1998; 339: 1349-1357.

32 Downs JR, Clearfield M, Weis S, et al. Primary prevention of acute coronary events with lovastatin in men and women with average cholesterol levels: results of AFCAPS/TexCAPS. Air Force/Texas Coronary Atherosclerosisi Prevention Study. JAMA 1998; 279: 1615-1622.

33 Shepherd J, Blauw GJ, Murphy MB, et al. Pravastatin in elderly individuals at risk of vascular disease (PROSPER): a randomised controlled trial. PROSPER study group. Prospective Study of Pravastatin in the Elderly at Risk. Lancet 2002; 360: 1623-1630.

34 Heart Protection Study Collaborative Group. MRC/BHF heart Protection Study of antioxidant vitamin supplementation in 20,536 high-risk individuals: a randomised placebo-controlled trial. Lancet 2002; 360: 23-33.

35 Sever PS, Dahlof B, Poulter NR, et al. ASCOT investigators. Prevention of coronary and stroke events with atorvastatin in hypertensive patients who have average or lower than average cholesterol concentrations, in the Anglo-Scandinavian Cardiac Outcomes Trial-Lipid-Lowering Arm (ASCOT-LLA): a multicenter randomised controlled trial. Lancet 2003; 361: 1129-1158.

36 Kastelein JJP, Bots ML. Statin therapy with ezetimibe or niacin in high-risk patients. $N$ Engl $J$ Med 2009; 361: 2180-2183.

37 Schwartz G, Olsson A, Ezekowitz M, et al. Effects of atorvastatin on early recurrent ischemic events in acute coronary syndromes: the MIRACL study: a randomized controlled trial. JAMA 2001; 285: 1711-1718.

38 Ray JG, Mamdani M, Tsuyuki RT, et al. Use of statins and the subsequent development of deep vein thrombosis. Arch Intern Med. 2001; 161: 1405- 1410.

39 Jick H, Zornberg GL, Jick SS, et al. Statins and the risk of dementia. Lancet 2000; 356: 1627- 1631.

40 Yaffe K, Barrett-Connor E, Lin F, et al. Serum lipoprotein levels, statin use, and cognitive function in older women. Arch Neurol 2002; 59: 378-384.

41 Pasco JA, Kotowicz MA, Henry MJ, et al. Statin use, bone mineral density, and fracture risk: Geelong Osteoporosis Study. Arch Intern Med 2002; 162: 537-540.

42 Collins R, Armitage J, Parish S, et al. Effects of cholesterol lowering with simvastatin on stroke and other major vascular events in 20,536 people with cerebrovascular disease or other high-risk conditions. Lancet 2004; 363: 757-767.

43 Buemi M, Senatore M, Corica F, et al. Stains and progressive renal disease. Med Res Rev 2002; 22: 76-84.

44 Athyros VG, Mikhailidis DP, Papageorgiou AA, et al. The effects of statins versus untreated dyslipidaemia on renal function in patients with coronary heart disease. A subgroup analysis of the Greek atorvastatin and coronary heart disease evaluation (GREACE) study. J Clin Pathol 2004; 57: 728-734.

45 Ose L, Davidson MH, Stein EA, et al. Lipid-altering efficacy and safety of simvastatin $80 \mathrm{mg}$ /day: long-term experience in a large group of patients with hepercholesterolemia. Worldwide Expanded Dose Simvastatin Study Group. Clin Cardiol 2000; 23: 39-46.

46 Gaist D, Rodriguez LA, Huerta C. Lipid-lowering drugs and risk of myopathy: a population-based follow-up study. Epidemiology 2001; 12: 565-569.

47 Jones PH, Davidson MH, Stein EA, et al. Comparison of the efficacy and safety of rosuvastatin versus atorvastatin, simvastatin, and pravastatin across doses (STELLAR trial). Am J Cardiol 2003; 92: 152-160.

48 Farmer JA, Torre-Amione G. Comparative tolerability of the HMG-CoA reductase inhibitors. Drug Saf 2000; 23: 197213.

49 Scott R, O'Brien R, Fulcher G, et al. Effects of fenofibrate treatment on cardiovascular disease risk in 9,795 individuals with type 2 diabetes and various components of the metabolic syndrome: the Fenofibrate Intervention and Event Lowering in Diabetes (FIELD) study. Diabetes Care 2009; 32: 493-498.

50 Farmer JA. Handbook of Cholesterol Management; Science Press: Marrickville, Austrilia, 2004; 74-75.

51 Neve BP, Fruchart JC, Staels B. Role of the peroxisome proliferatior-activated receptors (PRAR) in atherosclerosis. Biochem Pharmacol 2000; 60: 1245-1250.

52 MacIsaac R, Jerums G. Treatment of dyslipidemia in the elderly. J Pharm Prac Res 2002; 32: 188-193.

53 Gotto AM. Contemporary Diagnosis and Management of Lipid Disorders, $2^{\text {nd }}$ Edition; Handbooks in Healthcare: 
Newtown, PA, United States, 2001.

54 Knapp RH. Drug treatment of lipid disorders. $N$ Engl J Med 1999; 341: 498-511.

55 Arnos WS. Pharmacologic therapy of lipid disorders in the elderly. Am J Geriatr Cardiol 2002; 11: 247-256.

56 A cooperative trial in the primary prevention of ischeamic heart disease using clofibrate. Report from the Committee of Principal Investigators. Br Heart J 1978; 40: 1069-1118.

57 Frick MH, Elo O, Haapa K, et al. Helsinki Heart Study: primary prevention trial with gemfibrozil in middle-aged men with dyslipidemia. Safety of treatment, changes in risk factors, and incidence of coronary heart disease. N Engl J Med 1987; 317: 1237-1245.

58 The BIP Study Group. Secondary prevention by raising HDL cholesterol and reducing triglycerides in patients with coronary artery disease: the Bezafibrate Infarction Prevention (BIP) study. Circulation 2000; 102: 21-27.

59 Robins HB Robins SJ, Collins D, et al. Gemfibrozil for the secondary prevention of coronary heart disease in men with low levels of high-density lipoprotein cholesterol. Veterans Affairs High-Density Lipoprotein Cholesterol Intervention Trial Study Group. N Engl J Med 1999; 341: 410-418.

60 Chapman MJ, Assmann G, Fruchart JC, et al. Raising high-density lipoprotein cholesterol with reduction of cardiovascular risk: the role of nicotinic acid-a position paper developed by the European Consensus Panel on HDL-C. Curr Med Res Opin 2004; 20: 1253-1268.

61 Danish J, Collins R, Peto R. Lipoprotein (a) and coronary heart disease: meta-analysis of prospective studies. Circulation 2000; 102: 1082-1085.

62 Bays HE, McGovern ME. Once-daily niacin extended release/lovastatin combination tablet has more favorable effects on lipoprotein particle size and subclass distribution, than atorvastatin and simvastatin. Prev Cardiol 2003; 6:
179-188.

63 Elam MB, Hunninghake DB, Davis KB, et al. Effect of niacin on lipid and lipoprotein levels and glycemic control in patients with diabetes and peripheral arterial disease: the ADMIT study: a randomized trial. Arterial Disease Multiople Intervention Trial. JAMA 2000; 284: 1263-1270 .

64 Parra JL, Reddy KR. Hepatotoxicity of hypolipidemic drugs. Clin Liver Dis 2003; 7: 415-433 .

65 Kastelein JJP, Akdim F, Stroes ESG, et al. Simvastatin with or without ezetimibe in familial hypercholesterolemia. $N$ Engl J Med 2008; 358: 1431-1443.

66 Simons L, Tonkon M, Masana L, et al. Effects of ezetimibe added to on-going statin therapy on the lipid profile of hypercholesterolemia patients with diabetes mellitus or metabolic syndrome. Curr Med Res Opin 2004; 20: 1437-1445.

67 Ballantyne CM, Houri J, Notarbartolo A, et al. Effects of ezetimibe coadministered with atorvastatin in 628 patients with primary hypercholesterolemia: a prospective, randomized, double-blind trial. Circulation 2003; 107: 2409-2415.

68 Sager PT, Melani L, Lipka L, et al. Effect of coadministration of ezetimibe and simvastatin on high-sensitivity C-reactive protein. Am J Cardiol 2003; 92: 1414-1418.

69 Prosser LA, Stinnett AA, Goldman PA, et al. Costeffectiveness of cholesterol-lowering therapies according to selected patients' characteristics. Ann Intern Med 2000; 132: 769-779.

70 Ganz DA, Kuntz KM, Jacobson GA, et al. Costeffectiveness of 3-hydrosy-3-methylglutary coenzyme A reductase inhibitor therapy in older patients with myocardial infarction. Ann Intern Med 2000; 132: 780-787.

71 Cannon $\mathrm{CP}$, Braunwald $\mathrm{E}$, McCabe $\mathrm{CH}$, et al. Intensive versus moderate lipid-lowering with statins after acute coronary syndromes. N Engl J Med 2004; 350: 1495-1504. 